\title{
A propos de gel de conduites forcées
}

\section{The problem of freezing in penstocks}

\author{
PAR G. FERRAND
}

PRÉSIDENT-DIREcteur GÉNÉral dE LA SOCIÉTÉ DAUPHINOISE D'ÉTUdES LT de MONTAGES

\begin{abstract}
Caractère « impensable» du gel d'une conduite forcée. - Sévérité des consignes de sécurité dans ce domaine. - Etude du phénomène dans deux hypothèses différentes : glaciation en bloc sur une certaine longueur; glaciation progressive sans accroissement de volume, l'eau en excès étant chassée ver's l'aval. - Calcul des tensions résultantes qui, dans les denx cas, révèlent l'impossibilité industrielle d'éviter le dépassement de la limite élastique de la paroi de la conduite. - Intérêt des tuyaux surpressés, seuls da même de supporter sans rupture de telles déformations.
\end{abstract}

Une conduite forcée peut-elle geler? Si la chose s'est déjà produite, très rarement il est vrai, et sur d'anciennes conduites de petits diamètres en des circonstances tout à fait exceptionnelles, il est impossible de penser que cela puisse se produire maintenant, surtout lorsqu'il s'agit de conduites forcées modernes de grande puissance.

Non seulement une conduite forcée ne doit pas geler, mais toute formation d'un simple anneau de glace est interdite. Le gel complet d'un tuyau amène inévitablement sa rupture et la formation d'un anneau de glace constitue un grave danger pour les turbines lorsque, au dégel, des fragments de glace se détachent de la paroi de la conduite pour pénétrer dans les turbines.

Des dispositions sont done prises pour éviter toute formation de glace à l'intérieur des conduites forcées. Des consignes d'exploitation basées sur les études faites par M. Massé sur les conduites forcées du lac d'Oo, en 1938-39, sont partout en vigueur dans ce but. Nous les reproduisons ci-dessous :
The impossibility of a penstock freezing. The strict nature of safety precautions to be observed in this field. The phenomenon studied making two different assumptions: solid freezing over a certain lenglh, progressive freezing without an increase in bolume, excess water being expelled downstream. The resultant forces calculated demonstrating that it is industrially impossible to avoid exceeding the elastic limit of the penstock walls. The importance of using prestressed pipes which are capable of withstanding such deformation withoul fracturing.

\section{DISPOSITIONS ET PROTECTION CONTRE LE GRL}

«Dans les conduites, l'eau peut geler, si la vitesse admise est insuffisante. La vitesse minimum à admettre doit être déterminée par l'exploitant, compte tenu de la température minimum, de la situation de la conduite et des dispositions d'ensemble de l'aménagement hydraulique.

"Pour déterminer le débit minimum à conserver pendant les périodes de grands froids, on divise la conduite en troncons tels que, pour chacun, on puisse considérer comme constant le débit $Q$, la température ambiante $T$, la section $S$, le coefficient de transmission $K$ entre paroi et air ambiant.

«Appelons pour chaque troncon :

$\theta_{\text {II }}$ la température de l'eau au départ du tronçon,

$\theta_{\mathrm{N}}$ la température de l'eau au lerminus du troncon: posons :

$$
\theta_{0}=\theta_{\mathrm{I}}-\mathrm{T} \quad 0=\theta_{\mathrm{H}}-\mathrm{T}
$$


on obtient :

$$
{ }_{0}^{0_{0}}=e^{\mathrm{sK} / \mathrm{CQ}}
$$

dans laquelle :

e la base des logarithmes népériens $\log _{e}=0,434$,

S la surface totale de paroi du tronçon exposé à l'air,

C le coefficient calorifique de l'eau $=\sim 1.000$ calories par $\mathrm{m}^{3}$.

« Le volume du débit correspondant à 0 est donné par la formule suivante :

$$
\mathrm{Q}=\frac{\mathrm{SK}}{\mathrm{C}} \log e \frac{1}{\log \theta_{0}-\log \theta}
$$

$\mathbf{K}$ est à déduire d'essais à faire pour chaque cas particulier.

« On peut admettre pour $K$ maximum :

$$
\begin{gathered}
\frac{35}{3600} \\
\text { et pour K minimum }: \frac{10}{3600}
\end{gathered}
$$

«A la mise en marche de l'exploitation, et avant que l'exploitant ait pu déterminer la valeur réelle de $K$, il est recommandé de maintenir dans les conduites, pour mettre celles-ci à l'abri du gel, les vitesses suivantes:

Vitesse minimum

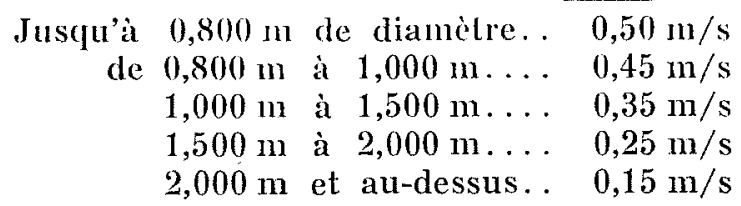

«En cas d'impossibilité de maintenir le débit nécessaire, soit par les turbines, soit par les robinets de vidange, les conduites seront complètement vidangées et l'exploitation arrêtée.

"Le gel n'est pas à craindre dans les conduites en puits ou en galeries fermées on enterrées et recouvertes sur un mètre d'épaisseur au-dessus de la génératrice supérieure.

«Les parties de conduites et accessoires où normalement l'eau est immobile et qui ne sont pas suffisamment abritées seront calorifugées.

« Les parties enrobées d'une épaisseur de un mètre de maconnerie sont à l'abri du gel.
«En ce qui concerne les collecteurs, des dispositions spéciales seront prises dans les périodes de grands froids, lorsque le débit est insuffisant pour alimenter toutes les turbines, et que, dans certaines parties, l'eau est immobile ou en mouvement ralenti. Par exemple, on procéderait aux calorifugeages nécessaires et on ferait fonctionner les turbines le plus à l'aval, à l'exclusion des autres.

"Lor'sque certaines parties des conduites et accessoires sont situées à l'intérieur de chambres de manœuvres, il est recommandé de chauffer celles-ci lors des périodes de grands froids, non seulement pour éviter le gel de l'eau, mais aussi pour assurer, le cas échéant, un fonctionnement normal des appareils de manouvre et de sécurité. $\gg$

Mais si le gel d'une conduite est impensable, il ne nous a pas paru inutile d'examiner ce qui pourrait se passer si l'eau d'une conduite forcée se gelait complètement.

On peut faire deux hypothèses très différentes sur le processus de glaciation totale d'une partie de la conduite.

I. - Sur une certaine longueur $L$, le poids total de la glace est le poids de l'eau correspondant au volume total de cette longueur.

II. - Avant que la prise soit complète, les aiguilles de glace ont laissé s'écouler, vers l'extrémité aval, une partic de l'eau non encore gelée.

L'hypothèse I correspond au cas d'un réservoir cylindrique, fermé aux extrémités, indéformable en longueur.

L'hypothèse II peut se traduire ainsi : pendant toute la durée de glaciation à $0^{\circ}$, l'accroissement de volume a été nul, la glace chassant l'eau en excès, de sorte que la pression est restée égale à la pression atmosphérique.

M. et Mme Chenars ont donné dans leur Jivre, Constantes physiques de l'eau, un diagramme représentant la diminution de volume, par fusion de la glace, à des températures comprises entre $0^{\circ}$ et $-20^{\circ}$; chacune de ces fusions étant obtenue par compression uniforme sous une pression qui est celle résultant de la formule de Clapeyron. Nous reviendrons plus loin sur celle-ci.

Chacune de ces transformations, efrectuce ainsi, étant réversible, nous pouvons considérer ce diagramme comme représentant tout aussi bien l'augmentation de volume d'un poids d'eau unití devenue slace et refroidic à - $0^{\circ}$, à la pression atmosphérique. Toulefois, en raisonnant ainsi, nous négligeons la variation de volume 
résultant, pour l'eau, de l'abaissement de température $\theta$ combiné à un accroissement de pression telle que l'état liquide soit maintenu jusqu'à $-0^{\circ}$. La quantité ainsi négligée est infime en regard du changement de volume de la transformation eau-glace.

\section{Nommons :}

Vo le volume initial à $0^{\circ}$ d'un poids $\pi$ d'eau à la pression atmosphérique,

$\Delta$ Vo l'augmentation de volume de cette eau transformée en glace, refroidie à -- $0^{\circ}$ sans changement de pression.

Le diagramme ci-dessus se traduit ainsi :

$$
\frac{\Delta \mathrm{V}_{0}}{\pi}=0,09+0,00210
$$

Le premier terme 0,09 provient de ce que à $0^{\circ}$, l'augmentation par glaciation est de $90 \mathrm{~cm}^{3}$ par $\mathrm{kg}$ d'eau.

\section{Hypothèse 1}

Le poids total est resté constant de $0^{\circ}$ à - $\theta$; mais l'augmentation de volume a été freinée par la paroi de la conduite. La pression, nulle lorsque l'eau était à $0^{\circ}$, est devenue $p$ à - $\theta^{\circ}$.

Nommons $k$ le coefficient de compressibilité de la glace.

Sous l'effet d'une pression uniforme $p$ dans toute la masse, le volume n'a pu augmenter de $\Delta V_{0}$, comme formule (1); il est devenu :

$$
V_{0}+\Delta V_{0}-k p\left(V_{0}+\Delta V_{0}\right)
$$

Nommons le $\mathrm{V}(\theta)$.

La propagation uniforme de la pression $p$ à la paroi ne pourrait pas être admise si la glace était un solide parfaitement élastique.

L’ouvrage précité montre que sa limite élastique est très faible $\left(\sim 0,3 \mathrm{~kg} \mathrm{~cm}^{2}\right)$ et que sa plasticité la rend coulante sous la pression. On peut done admettre qu'après un certain temps 0 et $p$ sont uniformes dans toute la masse.

On a finalement, au moyen de (1) et (2):

$$
V_{0}=V_{0}-k p V_{0}+\frac{\pi}{1000}(90+2,1 \theta)(1-k p)
$$

d'où :

$$
\frac{\mathrm{V} \theta-\mathrm{V}_{0}}{\mathrm{~V}_{\mathrm{O}}}=\frac{\pi}{1000 \mathrm{Vo}_{0}}(90+2,10)(1-k p)-k p
$$

En négligeant la petite difrérence de volume specifique de l'eau entre $4^{\circ}$ et $0^{\circ}$, on aura pour $\mathrm{L}=1 \mathrm{~cm}$, en nommant $r$ le rayon intérieur de la conduite et or l'augmentation de longueur du rayon correspondant à $\left(\mathrm{VO}_{\mathrm{O}}-\mathrm{VO}\right)$ :

$$
\begin{gathered}
V_{0}=\pi r^{2} \times(1 \mathrm{~cm}) \\
V_{0} 0-V_{0}=2 \pi r^{\circ} \times(1 \mathrm{~cm})
\end{gathered}
$$

d'où :

$$
\frac{2 \delta r}{r}=\frac{V 0-V_{0}}{V_{0}}=\frac{\pi}{1000 V_{0}}(90+2,1 \theta)(1-k p)-k p
$$

D'autre part, si la paroi s'était contractée librement du fait de l'abaissement de température de $0^{\prime \prime}$ à $-0^{\circ}$, son rayon serait devenu :

$$
r_{1}=r(1-\alpha \theta), \alpha \text { coefficient de dilatation de l'acier }
$$

La tension $t$, provenant de $p$, augmentera $r_{1}$ d'une quantité $d r_{1}$, telle que :

$$
\frac{d r_{1}}{r_{1}}=\frac{t}{\mathrm{E}}
$$

E module d'élasticité de l'acier. 
$r_{1}$ est done devenu, vu (4) et (5):

$$
r_{1}\left(1+\frac{d r}{r_{1}}\right)=r(1-a \theta)\left(1+\frac{t}{\mathrm{E}}\right)
$$

L'augmentation relative du rayon $r$ est :

$$
\frac{r(1-\alpha 0)[1+(t / \mathrm{E})]-r}{r}=\frac{t}{\mathrm{E}}-\alpha 0\left(1+\frac{t}{\mathrm{E}}\right)
$$

Mais elle est aussi $\partial r / r$ résultant de (3).

En igalant ces deux expressions de ôr $/ r$, il vient :

$$
\frac{\pi}{1000 \mathrm{Vo}}(90+2,10)(1-k p)-k p=2\left[-\alpha \theta\left(1+\frac{t}{\mathrm{E}}\right)+\frac{t}{\mathrm{E}}\right]
$$

Cette formule s'écrit :

$$
\left[1+\frac{\pi}{1000 \mathrm{Vo}_{0}}(90+2,10)\right] k p+2 \frac{t}{\mathrm{E}}(1-\propto 0)=\frac{\pi}{1000 \mathrm{Vo}_{0}}(90+2,10)+2 x_{0}
$$

La formule d'équilibre de la paroi donne :

$$
t=\frac{r}{e} p
$$

Portant dans la formule précédente, on aura :

$$
\left[\left(1+\frac{(90+2,10) \pi}{1000 \mathrm{VO}}\right) k+2 \frac{r}{e}(1-\alpha 0) \frac{1}{\mathrm{E}}\right] p=\frac{\pi}{1000 \mathrm{VO}}(90+2,10)+2 \times 0
$$

Dans ces formules :

$$
\left\{\begin{array}{l}
{ }^{a}=0,125 \cdot 10^{-4} \quad k=12 \cdot 10^{-\tau} \mathrm{cm}^{2} / \mathrm{kg} \\
\mathrm{E}=2000000 \mathrm{~kg} / \mathrm{cm}^{2}
\end{array}\right.
$$

Vo étant le volume d'une longueur de conduite de $1 \mathrm{~cm}$. Pour l'eau limpide, on a approximativement :

$$
\frac{\pi}{V_{0}}=1
$$

La formule précédente devient, en remplaçant $\alpha, k, \mathrm{E}, \pi / \mathrm{V}_{\mathrm{O}}$ par ces valeurs :

$$
\begin{gathered}
{\left[(1090+2,1 \theta) 12 \cdot 10^{-i}+10^{-3} \frac{r}{e}\left(1-0,125 \cdot 10^{-4} 0\right)\right] p=90+2,125 \theta} \\
p=\frac{90+2,125 \theta}{12 \cdot 10^{-\tau}(1090+2,1 \theta)+10^{-3}(r / e)\left(1-0,12510^{-4} \theta\right)}
\end{gathered}
$$

En laissant « $t$ » en évidence dans (6), on aurait obtenu, pour $p$, la formule :

$$
p=\frac{90-2000(t / \mathrm{E})+[2,1+2000 \alpha[1+(t / \mathrm{E})]]^{0}}{(1090+2,1) k}
$$

Voyons quelle valeur de $p$ donnent ces formules pour le moment où, à $0^{\circ}$, la prise en glace est achevée, e'est-à-dire en $\mathrm{y}$ faisant, $0=0$. II vient :

$$
\begin{aligned}
& p=\frac{90}{12.10^{-7} 1090+10^{-3}(r / e)} \\
& p=\frac{90-2000(t / \mathrm{E})}{(1090+2,1) 12.10^{-7}}
\end{aligned}
$$


Prenons pour $t$ le maximum qu'on puisse alteindre sans déformation permanente avec un luyau surpressć : $t=50 \mathrm{~kg} / \mathrm{mm}^{2}$, done 5000 par $\mathrm{cm}^{2}$.

La dernière $\left(10^{\prime}\right)$ donne :

$$
p=\frac{90-5000 \cdot 10^{-3}}{1092.12 .10^{-i}}=\frac{85}{1.31 .10^{-3}}=65000
$$

Celle valeur de $p$, portée dans (7), donne:

$$
t=\frac{r}{e} \times 650
$$

I'où :

$$
\frac{r}{t^{2}}=\frac{5000}{650}=\bar{i}, \bar{i}
$$

(c) qui n'est pas industriel.

L'autre formule donne:

$$
\begin{gathered}
p=\frac{90.10^{*}}{1,31+(r / e)} \\
\text { pour } \frac{r}{e}=100, \quad p=\frac{90.10^{3}}{101,3}=\sim 900
\end{gathered}
$$

Pour ce $r / e$ (7) donne $t=100900$. Done incompatibilité.

\section{CONCLUSIONS POUR L'HYPOTHÈSE I}

On peut en conclure que, dans l'hypothèse I, le gel complet à $0^{\circ}$ produit forcément des déformations permanentes $\left(^{*}\right)$. Ceci était d'ailleurs évident à priori. En effet, à $0^{\circ}$, l'ordre de grandeur des augmentations de volume du fait de la glaciation est $10 \%$, alors que le coefficient de compressibilité de la glace étant de l'ordre de 10-i, une augmentation de pression de $100 \mathrm{~kg}$ par $\mathrm{cm}^{2}$ diminue l'unité de volume de glace de $10^{-t}$ unités de volume seulement.

L'élasticité de la paroi est donc impuissante à empêcher le gonflement de glaciation à 0".

\section{Hypothèse II}

Après glaciation à $0^{\circ}$, le poids $\pi$ de glace contenu dans le volume vo de la conduite est inférieur de $90 \%$ au poids d'eau initial, puisque, comme on l'a vu, $90 \mathrm{~cm}^{3}$ d'eau par $\mathrm{kg}$ d'eau ont élé chassés vers l'aval au cours de cette glaciation.

On a donc :

$$
\pi=V_{0}-90 \frac{V_{0}}{1000}=V_{0}(1-0,09)
$$

D'autre part, le terme 0,09 de la formule (1) ayant disparu, celle-ci devient :

$$
\frac{\Delta V_{0}}{\pi}=0,00210
$$

(2) étant uniquement la traduction, en lettres, de la définition de $k$, elle est la même ici.

En portant dans (2) l'expression (12) de $\Delta V_{0}$, il vient:

$$
\mathrm{V} \theta=\mathrm{V}_{0}-k p \mathrm{~V}_{0}+(1-k p) \pi 0,00210
$$

${ }^{\star}$ ) Nous n’avons pas tenu compte, ici, que l'augmentation de la pression provenant de la ténacité de la paroi peut empêcher, en partie, la congélation; si les gouttes d'eau reparaissent, elles fuient sous la compression; on se trouve alors dans le cas de l'hypothèse II qui va ètre étudiéc ci-ap rès. 
ou bien :

$$
\frac{\mathrm{V}_{\theta}-\mathrm{V}_{0}}{\mathrm{~V}_{0}}=\frac{\pi}{1000 \mathrm{Vo}_{0}} 2,1 . \theta(1-k p)-k p
$$

En tenant compte de (11):

$$
\frac{V_{\theta}-V_{0}}{V_{0}}=\frac{1-0,09}{1000} 2,1 \theta(1-k p)-k p
$$

(3) devient alors :

$$
\frac{2 \delta r}{r}=\frac{V \theta-V_{0}}{V_{0}}=\frac{1-0,09}{1000} 2,1 \theta(1-k p)-k p
$$

L'expression de $\delta r / r$ provenant de $t$ et de $\theta$ est, comme précédemment :

$$
\frac{t}{\mathrm{E}}-\alpha \theta\left(1+\frac{t}{\mathrm{E}}\right)
$$

de sorte que :

$$
\frac{1-0,09}{1000} 2,1 \theta(1-k p)-k p=2\left[\frac{t}{\mathrm{E}}-\alpha \theta\left(1+\frac{t}{\mathrm{E}}\right)\right]
$$

Cette relation s'écrit :

ou bien :

$$
1,911 \theta(1-k p)-1000 k p=2000\left[(1-\alpha 0) \frac{t}{\mathrm{E}}-\alpha \theta\right]
$$

$$
(1,911+2000 \alpha) \theta=2000 \frac{t}{\mathrm{E}}(1-\alpha \theta)+(1000+1,9110) k p
$$

En portant dans cette expression les valeurs numériques (8), on obtient :

$$
1,9360=\left(1-0,125 \cdot 10^{-4} \theta\right) \frac{t}{1000}+12 \cdot 10^{-\tau}\left(1000+1,911^{\theta}\right) p
$$

en utilisant (7), ceci devient :

$$
1,9360=\left[\left(1-0,125 \cdot 10^{-4} \theta\right) \frac{1}{1000} \frac{r}{e}+12.10^{-7}(1000+1,911 \theta)\right] p
$$

(13) et (14) donnent comme expressions de $p$ :

$$
\begin{gathered}
p=\frac{-(t / 1000)+\left[1,936+0,125 \cdot 10^{-7} t\right] \theta}{12.10^{-\tau}(1000+1,911 \theta)} \\
p=\frac{1,936 \theta}{12.10^{-7}(1000+1,911 \theta)+(1 / 1000) r / e\left(1-0,125.10^{-4} \theta\right)}
\end{gathered}
$$

Abaissement de la température de fusion du fatt de la compression

On connaît la formule de Clapeyron :

$$
\lambda=\mathrm{T}\left(v^{\prime}-v\right) \frac{d p}{d \theta}
$$

où :

$\lambda$ est la chaleur latente de fusion,

$v^{\prime}$ volume spécifique de l'eau, $v$ volume spécifique de la glace (à $\mathrm{t}^{\circ}$ sous une pression $p$ ),

T température absolue de fusion $=\theta+273$.

Au voisinage de $0^{\circ}$, cette formule donne, si ta pression est exprimée en atmosphères :

$$
\frac{d \theta}{d p}=-0,0075
$$


Comme $0=0$, pour $p=1$ atm., il en résulte :

$$
p=1-\frac{0}{0,0075}
$$

oll :

$$
p=1-133,40
$$

Celte formule donne la table suivante :

Température

de fusion : $0^{\circ}-1^{\circ}-2^{\circ} 5-5^{\circ}-7^{\circ} 5$

Pression nécessaire :

(en atmosphères) $1133 \quad 333 \quad 666 \quad 1000$

Etant donné le degré d'approximation de tout ceci, nous remplacerons 1 atm. par $1 \mathrm{~kg} / \mathrm{cm}^{2}$.

Exempres : Voyons si à -1" la fusion de la slace pourra se produire avant que la tension de la paroi ait atteint la limite élastique.

Pour cela, faisons $0=1, p=133$ (voir 15'), dans la formule (13). On obtient :

$\left(\begin{array}{lll}1-0,125 & \left.10^{-4}\right) t\end{array}\right.$

$$
=1000\left(1,936-12 \cdot 10^{-\tau} 1001,911\right) 133
$$

d'où :

$$
t=1776 \mathrm{~kg} / \mathrm{cm}^{2}
$$

(7) donne :

$$
\frac{r}{e}=\frac{1776}{133}=13,35
$$

Pour $-2^{\circ}$, d'où $p=266$, on trouve :

$$
t=3553, \text { d'où }(r / e)=13,36
$$

Pour $-5^{\circ}$, d'où $p=666$, on tronve :

$$
t=8870, \text { d'où }(r / e)=13,3
$$

Essayons $(r / e)=100$ et voyons s'il peut y avoir fusion de la glace par compression venant de la paroi.

Utilisons la formule $\left(14^{\prime}\right)$; voyons ce qu'elle donne, pour $(r / e)=100$ all diverses températures. On obtient :

Pour $0=1 p=19,36,(7)$ donne alors $t=1936$.
Pour $0=2 p=38,72$, (7) donne alors $t=3872$.

A plus basse température on dépasserail la limite élastique pour $t$.

Dans les deux cas nous voyons que la limite élastique est atteinte bien avant que la fusion se produise (Lableau 15').

Pour $(r / e)>100$, la même formule montre que la limite élastique est atteinte pour des températures comprises entre 0 et $-2^{\circ}$.

Cherchons maintenant, au moyen de la formule $\left(13^{\prime}\right)$, à quelle température sera atteinte une tension maximum de $35 \mathrm{lg} / \mathrm{mm}^{2}$, elle montre qu'il faut descendre plus bas que -2 " :

$$
\begin{aligned}
& \text { à - } 2^{\circ}, \text { on aura } p=230 \\
& \text { à }-3^{\circ}, \text { on aura } p=1920
\end{aligned}
$$

Au-delà de - 2", on dépasse done la pression résultant de la formule (15) et du tableau (15').

Mais la formule (7) montre que, au-delà de - 2", les $(r / e)$ correspondant aux $\left\langle p »\right.$ de $\left(15^{\prime}\right)$ sont voisins de 13,3 . On retombe sur le cas du premier exemple de ce paragraphe.

\section{Conclusions pour l'hypothise II}

La surfusion par compression ne peut se produire dans les conduites forcées.

La limite élastique de la paroi est atteinte pour des températures voisines de $-2^{\circ}$ quand $r_{\prime}^{\prime} e$ est voisin de 100.

Pour des parois de plus faible épaisseur, elle est atteinte pour des températures se rapprochant de plus en plus de $0^{\circ}$.

Ainsi done le gel d'une conduite amènerait inévitablement la déformation plastiçue des tuyaux avec risque de rupture pour ceux dont l'épreuve hydraulique n'aurait pas été pousséc jusqu'à obtenir et dépasser la limite ćlastique apparente de l'acier les constituant. Par contre, les tuyaux surpressés qui, par définition, subissent au cours de leur fabrication une déformation plastique importante, subiraient sans risque de rupture une déformation supplémentaire, car les possibilités de déformations restantes des tuyaux surpressés avant rupture sont encore supérieures à celles que pourrait amener le gel. 